\title{
Hippocampal Functional Connectivity and Memory Performance After Exercise Intervention in Older Adults with Mild Cognitive Impairment
}

\author{
Junyeon Won ${ }^{\mathrm{a}}$, Daniel D. Callow ${ }^{\mathrm{a}, \mathrm{b}}$, Gabriel S. Pena ${ }^{\mathrm{a}}$, Leslie S. Jordan ${ }^{\mathrm{a}, \mathrm{b}}$, \\ Naomi A. Arnold-Nedimala ${ }^{\mathrm{a}}$, Kristy A. Nielson ${ }^{\mathrm{c}, \mathrm{d}}$ and J. Carson Smith ${ }^{\mathrm{a}, \mathrm{b}, *}$ \\ ${ }^{a}$ Department of Kinesiology, University of Maryland, College Park, MD, USA \\ ${ }^{\mathrm{b}}$ Program in Neuroscience and Cognitive Science, University of Maryland, College Park, MD, USA \\ ${ }^{\mathrm{c}}$ Department of Psychology, Marquette University, Milwaukee, WI, USA \\ ${ }^{\mathrm{d}}$ Department of Neuropsychology, Medical College of Wisconsin, Milwaukee, WI, USA
}

Accepted 10 May 2021

Pre-press 11 June 2021

\begin{abstract}
.
Background: Exercise training (ET) has neuroprotective effects in the hippocampus, a key brain region for memory that is vulnerable to age-related dysfunction.

Objective: We investigated the effects of ET on functional connectivity (FC) of the hippocampus in older adults with mild cognitive impairment (MCI) and a cognitively normal $(\mathrm{CN})$ control group. We also assessed whether the ET-induced changes in hippocampal FC ( $\Delta$ hippocampal-FC) are associated with changes in memory task performance ( $\Delta$ memory performance). Methods: 32 older adults $(77.0 \pm 7.6$ years; $16 \mathrm{MCI}$ and $16 \mathrm{CN})$ participated in the present study. Cardiorespiratory fitness tests, memory tasks (Rey Auditory Verbal Learning Test (RAVLT) and Logical Memory Test (LM)), and resting-state fMRI were administered before and after a 12-week walking ET intervention. We utilized a seed-based correlation analysis using the bilateral anterior and posterior hippocampi as priori seed regions of interest. The associations of residualized ET-induced $\Delta$ hippocampal-FC and $\Delta$ memory performance were assessed using linear regression.

Results: There were significant improvements in RAVLT Trial 1 and LM test performance after ET across participants. At baseline, MCI, compared to CN, demonstrated significantly lower posterior hippocampal FC. ET was associated with increased hippocampal FC across groups. Greater ET-related anterior and posterior hippocampal FC with right posterior cingulate were associated with improved LM recognition performance in MCI participants.

Conclusion: Our findings indicate that hippocampal FC is significantly increased following 12-weeks of ET in older adults and, moreover, suggest that increased hippocampal FC may reflect neural network plasticity associated with ET-related improvements in memory performance in individuals diagnosed with MCI.
\end{abstract}

Keywords: Exercise training, functional connectivity, hippocampus, memory, mild cognitive impairment, older adults

\section{INTRODUCTION}

*Correspondence to: J. Carson Smith, PhD, Department of Kinesiology, University of Maryland, School of Public Health, 2351 SPH Bldg \#255, College Park, MD 20742, USA. Tel.: +1 301405 0344; Fax: +1 301405 5578; E-mail: carson@umd.edu.
Memory decline is one of the most common hallmarks of age-related cognitive impairment [1]. Age-related memory decline is closely linked to 
deteriorated hippocampal function, a brain region important for memory and significantly impacted by the pathological aging processes [2]. Indeed, volume atrophy [3], reduced resting blood flow [4], impaired synaptic plasticity [5], and mitochondrial dysfunction [6] have been observed in the aging hippocampus of humans and rodents. Severe memory deficits and compromised hippocampal function are key aspects of the clinical symptomatology of mild cognitive impairment (MCI), a prodromal stage of dementia characterized by memory loss in the absence of impairment in activities of independent daily living [7]. Hippocampal structural and functional deterioration (e.g., atrophy, neuronal cell loss, reduced synaptic density) [8] and associated memory deficits [9] are also pronounced in MCI. Given that 16 million American older adults are currently living with $\mathrm{MCI}$ [10] and MCI is a major risk factor for developing Alzheimer's disease (AD) [11], identifying effective and accessible interventions to address this public health concern is of paramount significance.

Aerobic exercise training (ET) is a prime candidate for treating the aging brain due to its well-established neuroprotective effects [12]. These effects are particularly evident in the hippocampus and memory tasks, as evidenced by increased anterior hippocampal volume and concomitantly enhanced spatial memory performance in healthy older adults after a 6-month ET [13]. Animal models delineate that ET improves learning during the Morris water maze task, a task highly dependent on spatial navigation that is mediated by the hippocampus, by promoting hippocampal neurogenesis (i.e., growth of new neurons) [14]. However, the demonstration of hippocampal neuroplasticity in individuals diagnosed with MCI has not been widely reported. Nagamatsu et al. (2013) observed improvements in spatial and verbal memory performance following a 6-month ET in MCI [15]. Meanwhile, older women with MCI who engaged in a 6-month aerobic ET maintained hippocampal volume compared to hippocampal volume loss in those who completed a balance and toning (BAT) intervention [16]. Taken together, these findings prompt the hypothesis that improvements in memory performance after ET in individuals diagnosed with MCI may involve alterations in network connectivity rather than volumetric growth of the hippocampus.

There is recent evidence in healthy older adults that ET alters the hippocampal functional connectivity network. Based on the coherence of functional magnetic resonance imaging (fMRI) blood oxygenation level-dependent (BOLD) signals [17], resting-state functional connectivity (FC) analysis is a noninvasive approach to understand the patterns of functional interaction between spatially remote brain regions [18]. Using a whole-brain correlation analysis with bilateral hippocampus as seed regions of interest, a 12-week Tai Chi intervention found a training-induced increase in hippocampal FC with regions in the medial prefrontal cortex in older adults, and a corresponding improvement in memory performance [19]. In a cross-sectional study of healthy younger adults (ages 20-38) that employed a whole-brain analysis (bilateral anterior and posterior hippocampal seeds), greater cardiorespiratory fitness (measured by $\dot{\mathrm{V}}_{2 \max }$ ) was associated with stronger FC between the bilateral anterior hippocampus and multiple brain regions including prefrontal and temporal cortical regions [20].

While there is evidence for ET-related alterations in hippocampal connectivity in healthy younger and older adults, it has not been conclusively established whether aerobic ET alters hippocampal functional networks in older adults diagnosed with MCI. A demonstration of ET-related hippocampal FC changes in MCI that co-occur with improvement in hippocampal-dependent task performance would provide evidence for hippocampal network plasticity and would have implications toward neuroprotective interventions in MCI. To address these gaps in the literature, we tested the effects of a 12-week walking intervention on hippocampal FC in cognitively normal (CN; control group) older adults and older adults diagnosed with MCI. In addition to the hippocampal seed region of interest, a bilateral amygdala seed, a nearby subcortical gray matter region in the medial temporal lobe, was included to test for the specificity of these effects. We also examined associations between changes in hippocampal FC and changes in memory performance after ET. Based on prior findings that demonstrated increased hippocampal FC using a whole-brain analysis (with hippocampal seeds) $[19,20]$ and our previous finding of ET-related increased compensatory response in neural networks in MCI [21], we hypothesized that ET would increase FC of the hippocampus both in CN older adults and those diagnosed with MCI. Secondly, we hypothesized that ET-related increases in hippocampal FC would be associated with improved memory performance in $\mathrm{CN}$ older adults and those diagnosed with MCI. Lastly, we hypothesized that ET would confer greater hippocampal FC and memory improvements in $\mathrm{MCI}$ compared to $\mathrm{CN}$. 


\section{METHODS}

\section{Participants}

Thirty-five community-dwelling older adults (17 MCI and 18 healthy older adults; ages 60-88) participated in this study. We used local newspaper advertisements, in-person informational sessions at local retirement communities, and physician referrals to recruit participants. Interested individuals were screened using a structured telephone interview to determine preliminary eligibility, including exclusionary health conditions and MRI contraindications. Final eligibility was determined after participants underwent a neurological assessment. Written consent form and physician approval for participating in moderate-intensity ET were obtained from qualified participants. This study was approved by the Institutional Review Board of the Medical College of Wisconsin in accordance with the Helsinki Declaration.

\section{Inclusion and exclusion criteria}

Physically inactive older individuals who engage in moderate-intensity physical activity less than 3 days/week for the past 6 months (self-reported) were included to the study. We previously reported a detailed description about the eligibility criteria and recruitment in the present study [22]. Briefly, individuals were considered ineligible if they reported: 1) neurological or cerebrovascular conditions (e.g., Parkinson's disease, Huntington's disease, multiple sclerosis, epilepsy, carotid artery disease, and brain tumor); 2) untreated Axis I psychiatric disturbance meeting DSM-IV Axis I criteria, severe depressive symptoms, and substance abuse; 3 ) history of cardiovascular disease or brain injury (e.g., stroke); 4) MR contraindications (e.g., metal implants, pacemakers, and a history of claustrophobia); 5) left-handedness (i.e., <50 of laterality quotient [23]); 6) history of ischemic attack; 7)>15 score on the 30-item Geriatric Depression Scale (GDS) [24] or relatively impaired activities of daily living [25].

\section{Neurocognitive testing}

Each participant's cognitive status was evaluated by a team of clinical investigators who made determinations based on the core clinical criteria for the diagnosis of MCI by National Institute of AgingAlzheimer's Association criteria for diagnosis of
MCI [26]. The following criteria were utilized to define MCI: 1) subjective cognition-related concerns; 2 ) impaired cognition ( $\geq 1$ cognitive domain); 3) preserved activities of daily living; 4) absence of dementia. Further examination was followed on individuals with probable MCI by a neurologist to exclude other possible causes of cognitive decline. All participants completed a full neuropsychological battery that assessed episodic memory, executive function, and processing speed.

\section{Memory tasks}

As part of a larger neuropsychological test battery, participants completed the Rey Auditory Verbal Memory Test (RAVLT) and Logical Memory (LM) subtest of the Wechsler Memory Scale-III before and following a 12-week ET. The tests were administered between $7 \mathrm{AM}$ and $11 \mathrm{AM}$. The average difference between the last day of ET intervention and postintervention test across all participants was $5.3 \pm 3.9$ days. The RAVLT is designed to assess aspects of both short-term memory and delayed recall [27]. During the five separate trials, an examiner reads a list of 15 unrelated words to the participant and asks them to repeat as many of these words as possible. After the fifth trial, an interference-list of 15 other words were read to the participants. After recall of the interference list, participants were asked to again spontaneously recall the original words (immediate recall). Finally, after a $20 \mathrm{~min}$ delay period, participants were instructed to spontaneously recall the original words (delayed recall). Including the delay period, the test lasted approximately $45 \mathrm{~min}$. The primary interpretive indices of the RAVLT were the number of words recalled at Trial 1, the total number of words recalled across Trials 1-5, immediate recall, delayed recall, and the number of correct responses in the recognition test. Alternate forms of the RAVLT were counterbalanced at the before and after ET sessions to minimize possible learning effects across participants.

The LM test is designed to assess verbal episodic memory for narratives [28]. Participants heard two short stories and were asked to immediately and completely recall them. Recall was tested again after a 30 min delay, followed by a yes/no recognition test of the story details (15 questions per narrative). The number of correct responses during the immediate recall, delayed recall, and recognition were used for the present study. 


\section{Cardiorespiratory fitness test}

All subjects performed a modified submaximal stress test to measure aerobic fitness before and after the 12-week exercise intervention. We utilized a modified Balke-Ware protocol (initial exercise speed $=3.2 \mathrm{~km} / \mathrm{h}$ at $0^{\circ}$ grade and grade increase $1 \% \mathrm{~min}$ ) on a General Electric (GE) motorized treadmill (Milwaukee, WI). Based on breath-by-breath calculations of the oxygen $\left(\mathrm{O}_{2}\right)$ and carbon dioxide $\left(\mathrm{CO}_{2}\right)$ content in expired air, measures of ventilation, rate of oxygen $\left(\mathrm{VO}_{2}\right)$ consumption, rate of carbon dioxide $\left(\mathrm{VCO}_{2}\right)$ production, and the respiratory exchange ratio (RER; $\mathrm{VCO}_{2} / \mathrm{VO}_{2}$ ) were obtained using a 15 -sec rolling average on a calibrated metabolic measurement system (Parvo Medics, Salt Lake City, UT). We recorded $\mathrm{VO}_{2}$ up to $85 \%$ of $\mathrm{HRR}$, and these data were used to estimate $\mathrm{VO}_{2 \text { peak }}$ [29]. To monitor subjective effort, we utilized the Borg rating of perceived exertion (RPE) scale [30], which was measured every min. Heart rate (HR) and blood pressure were measured every 2 min during the test. Heart rate reserve (HRR) was determined based on age-predicted maximal heart rate and resting heart rate measured while seated prior to the exercise test. The test was terminated upon reaching $85 \%$ of the participant's maximal HRR or upon observation of absolute exercise contraindications (e.g., raise in diastolic blood pressure $>110 \mathrm{mmHg}$ ), or participant's request to stop the test.

\section{Exercise intervention}

The study participants underwent a 12-week treadmill walking intervention (44 total sessions). All exercise sessions were supervised by a certified personal trainer or exercise physiologist. No more than two individuals participated in each exercise session. Each walking exercise session lasted for $30 \mathrm{~min}$ and was administered 4 sessions/week in local recreation centers. To assess exercise intensity, HR monitor (Polar Electro, Kempele, Finland) and RPE scale [30] were used during exercise, with the goal of achieving a moderate intensity during each session. The exercise session intensity, session duration, and weekly frequency were gradually increased during the first 4 weeks until participants were walking 30 min per session (4 sessions/week). During the 5-12 weeks, the exercise intensity was targeted at $50-60 \%$ of HRR and exercise duration was $30 \mathrm{~min}$. To tailor exercise intensity for each participant, the treadmill speed and grade were modified each session based on HR and
RPE based on individual baseline exercise capacity. A 10 min warm-up and 10 min cool-down consisted of light walking and flexibility exercise were administered before and after each session. Thus, total exercise session length was 50 min including warmup and cool-down.

\section{MRI acquisition}

All MRI data were acquired on a 3.0 Tesla GE (Waukesha, WI) MR scanner. A high-resolution T1-weighted anatomical image was acquired for co-registration with the following sequence parameters: 3D Spoiled Gradient Recalled at steady state protocol (SPGR), field of view $(F O V)=240 \mathrm{~mm}$, voxel size $=0.94 \times 0.94 \times 1.00 \mathrm{~mm}$, number of excitations $(\mathrm{NEX})=1$, slice thickness $=1 \mathrm{~mm}$, repetition time $(\mathrm{TR})=9.6 \mathrm{~ms}$, echo time $(\mathrm{TE})=3.9 \mathrm{~ms}$, inversion recovery preparation time $=450 \mathrm{~ms}$, flip angle $=12^{\circ}$, resolution $=256 \times 224$, and sequence duration $=6 \mathrm{~min}$. The resting state BOLD data were acquired using the following sequence parameters: gradient echo planar images, FOV $=240 \mathrm{~mm}$, $\mathrm{NEX}=1 \mathrm{~mm}$, slice thickness $=1.0 \mathrm{~mm}, \mathrm{TR} / \mathrm{TE}=$ $2000 / 25 \mathrm{~ms}$, axial slices $=36$, flip angle $=77^{\circ}$, resolution $=64 \times 64$, and sequence duration $=6 \mathrm{~min}$. During the resting state scan, participants were instructed to remain still and to keep their eyes on a fixation cross projected in the screen.

\section{Structural and functional MRI data processing}

The T1-weighted anatomical volumes were processed using FreeSurfer's (version 5.3.0) automated processing stream (recon-all) to generate cortical and subcortical reconstructions based on tissue-specific intensities and atlas probabilities [31]. Next, the first three volumes of the functional image time-series were manually removed to minimize magnetization disequilibrium using Analysis of Functional NeuroImage's [32] (AFNI, v.17.2.10) 3dTcat function. The truncated time-series were realigned using SliceOriented Motion Correction (SLOMOCO) which uses both in- and out-of-plane motion parameters to correct for misalignment between consecutive slices [33]. The motion-corrected functional volumes were then co-registered to FreeSurfer-rendered anatomical images using AFNI's align_epi_anat function and were visually inspected for proper alignment. The co-registered time-series were then entered into AFNI's single-subject preprocessing stream (proc.py). Volumes containing outlier fraction 
threshold $(>10 \%)$ were censored from the timeseries. The remaining volumes were despiked (3dDespike) to attenuate high-intensity transients within the BOLD signal and time-shifted to the beginning of the TR (3dTshift). Image volumes were also censored if their frame-wise displacement was greater than $0.2 \mathrm{~mm}$ to reduce the spurious effects of head motion. The anatomical image and anatomical followers (FreeSurfer-processed gray matter, white matter, and ventricular segmentations) were warped to the standard space (AFNI's MNI152_T1_2009c template) using non-linear transformation (3dQwarp). To further reduce physiological noise, nuisance physiological artifacts (e.g., motion derivatives and signals from ventricles and white matter) were eroded. The resolution of the resulting transformation matrix had a grid spacing of $2 \times 2 \times 2 \mathrm{~mm}^{3}$ and was used in the subsequent connectivity analyses. The effects of head movement did not confound the subsequent fMRI data interpretation because there was no difference in the percentage of censored TRs between the before $(0.86 \pm 0.27 \%)$ and after ET scans $(0.94 \pm 0.34 \%)(p=0.122)$.

\section{Hippocampal functional connectivity analysis}

We utilized a seed-based correlation analysis using the bilateral anterior and posterior hippocampi as priori seed regions of interest, based on prior works documenting stronger ET-related associations with the anterior part of the hippocampus relative to posterior regions [13, 20]. To accomplish this, left and right hippocampal masks were extracted from each participant's anatomical follower (Freesurfer-processed subcortical segmentation) that was warped to the standard MNI space [31]. Each of the hippocampal masks was then split into anterior and posterior parts based on the center of gravity of the region (Fig. 1). The bilateral anterior and posterior hippocampi masks were then combined using AFNI's $3 \mathrm{dcalc}$ function, respectively, and were used as primary seeds of interest. We also evaluated the effects of ET on the bilateral whole hippocampus using not divided hippocampal masks. Left and right amygdalar masks were also extracted from the anatomical follower, combined to create bilateral amygdalar mask using the $3 \mathrm{dcalc}$ function, and was used as control seed to test the specificity of ET-related FC changes. The final segmentations for each seed were visually inspected for quality assurance. The average signal time series were then extracted from each seed region and were cross-correlated with all voxels in

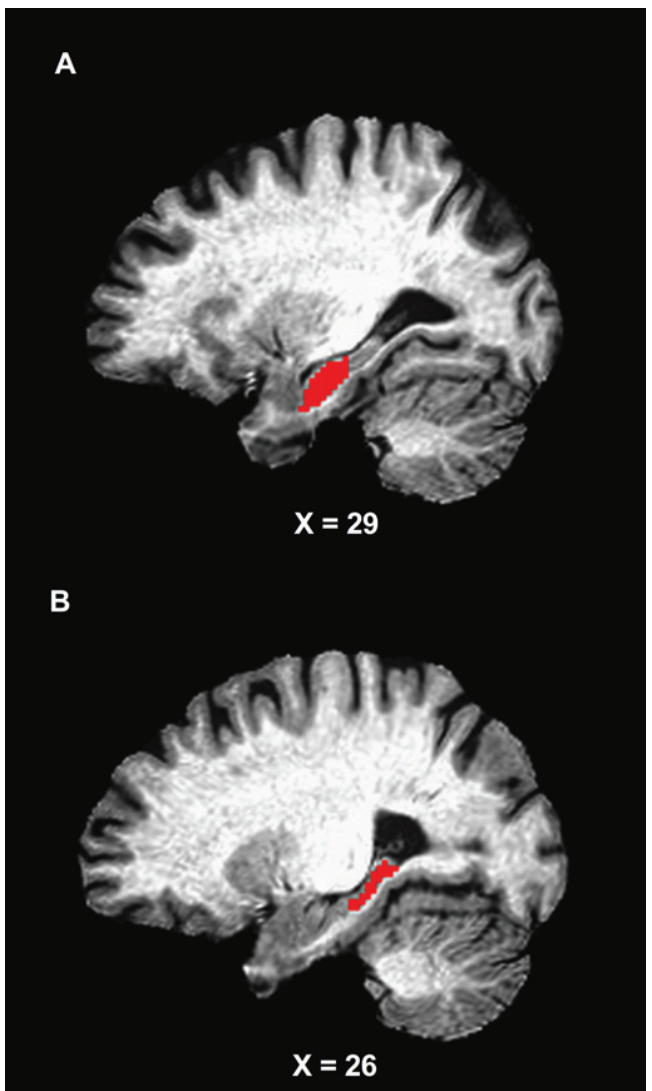

Fig. 1. Location of the anterior (A) and posterior (B) seed regions. The seed masks are exhibited on a representative subject's structural image.

the brain to isolate a brain FC map. These maps were created for each participant and each experimental time point (e.g., before and after ET). Finally, using a Fisher's r-to-z transformation, correlation coefficients were standardized for group-level analysis.

\section{Gray matter voxel-wise group analysis}

We created the whole-brain gray matter mask using FreeSurfer segmentation for each participant. White matter and ventricle segmentations were excluded. The gray matter mask was merged across participants for a group-level mask. Subject-level $z$-scored correlation maps were submitted to AFNI's linear mixed effects model (3dLME) to measure 1) changes in the anterior and posterior hippocampal connectivity from before to after ET (i.e., main effects of Time); 2) differences between groups (MCI versus $\mathrm{CN}$; main effects of Group); and 3) the Group $\times$ Time interaction. AFNI's cluster-size threshold computation program (3dClustSim) was used to control the whole 
Table 1

Demographic information for study participants

\begin{tabular}{|c|c|c|c|c|c|}
\hline & & $\begin{array}{l}\text { Total Sample } \\
\quad(n=32)\end{array}$ & $\begin{array}{c}\text { MCI } \\
(n=16)\end{array}$ & $\begin{array}{c}\mathrm{CN} \\
(n=16)\end{array}$ & $\begin{array}{c}\text { Group } \\
\text { Differences }\end{array}$ \\
\hline \multirow{6}{*}{ Demographics } & & Mean \pm SD & Mean \pm SD & Mean \pm SD & $p$ \\
\hline & & & & & \\
\hline & Age (y) & $77.0 \pm 7.6$ & $78.8 \pm 7.6$ & $75.3 \pm 7.4$ & 0.194 \\
\hline & Female $(n, \%)$ & $23(71.8 \%)$ & $10(43.5 \%)$ & $13(56.5 \%)$ & $0.220_{\mathrm{F}}$ \\
\hline & Education (y) & $15.8 \pm 2.2$ & $15.0 \pm 2.1$ & $16.6 \pm 2.0$ & 0.128 \\
\hline & $A P O E \& 4$ Carriers $(n, \%)$ & $12(37.5 \%)$ & $6(37.5 \%)$ & $6(37.5 \%)$ & \\
\hline Cardiorespiratory Fitness & Baseline $\dot{\mathrm{V}} \mathrm{O}$ & $190+43$ & $185+41$ & $106+20$ & 0444 \\
\hline \multirow[t]{2}{*}{ Depression } & 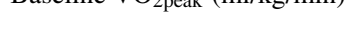 & & & & \\
\hline & Baseline GDS & $4.4 \pm 3.1$ & $5.6 \pm 3.6$ & $3.1 \pm 1.8$ & 0.056 \\
\hline \multirow[t]{2}{*}{ Cognitive Function } & & & & & \\
\hline & Baseline DRS-2 & $35.7 \pm 1.3$ & $35.3 \pm 1.6$ & $36.1 \pm 0.7$ & 0.079 \\
\hline Activities of Daily Living & Bassline Lawton ADL & $4.7 \pm 0.4$ & $4.6 \pm 0.4$ & $4.7 \pm 0.4$ & 0.780 \\
\hline
\end{tabular}

MCI, mild cognitive impairment; CN, normal cognition control; F, Fisher's Exact Test; APOE $\varepsilon 4$, apolipoprotein E epsilon 4 allele; $\dot{\mathrm{V}} \mathrm{O}_{2 \text { peak }}$,peak rate of oxygen consumption; GDS, Geriatric Depression Scale; DRS-2, Mattis Dementia Rating Scale-2; ADL, Activities of Daily Living.

brain family-wise error rate (FWER) at $p<0.05$ based on a voxel-wise probability threshold of $p<0.001$ and minimum cluster size of $k \geq 41\left(328 \mathrm{~mm}^{3}\right)$.

\section{Hippocampal volume assessment}

We assessed the hippocampal volume to examine whether ET-related changes in hippocampal FC occurred independent of those in hippocampal volume. The FreeSurfer-defined volumes of the hippocampus were calculated based on FreeSurfer's anatomical segmentation algorithm (recon-all) [31]. Subsequently, each participant's hippocampal volume was expressed relative to total intracranial volume (ICV) which was also calculated from the FreeSurfer algorithm. This adjustment was based on previous investigations that identified significant sex-related differences in ICV [34, 35]. Therefore, we used bilateral hippocampal volume/TIV as a measurement of hippocampal volume and computed the correlation between the ET-related changes hippocampal FC and baseline/ET-induced changes in hippocampal volume, respectively.

\section{Statistical analysis}

After determining normality using the ShapiroWilk test, the baseline demographic differences between groups (MCI versus $\mathrm{CN}$ ) were analyzed using independent sample $t$-tests (or Wilcoxon signed-rank tests) or Fisher's exact test. Normality of memory test performances was also tested using the Shapiro-Wilk test. Repeated measures ANOVA were used to compute the main effects of Time (i.e., before versus after ET), Group (i.e., MCI versus CN), and Group $\times$ Time interaction on the RAVLT and the LM performances. The association between ETinduced changes in memory performance ( $\Delta$ memory performance) and hippocampal FC ( $\Delta$ hippocampalFC) was then examined. To assess this, average $z$-scored connectivity within the anterior and posterior hippocampi, respectively, were extracted from subject-level correlation maps. We then used multivariable linear regression. Prior to this test, we conducted bivariate correlation tests to evaluate relationships between demographic characteristics (age, sex, education) and variables of interest ( $\Delta$ memory performance and $\Delta$ hippocampal-FC) in MCI group. Significant correlation between age and some memory task performances (RAVLT Trial 1, Trial 1-5, immediate recall) were found in $\mathrm{CN}$; thus, the correlation analyses were not adjusted for in the MCI group and age was controlled for in $\mathrm{CN}$ group.

Residualized FC and memory task performance were independently derived from linear regression models predicting post-intervention from baseline values. The residualized change scores were used to minimize both practice effects and regression to the mean [36, 37]. Next, residualized hippocampal FC and residualized memory performance were included as the independent and dependent variables, respectively. Lastly, we performed a post-hoc analysis to assess the interaction between Group (MCI versus $\mathrm{CN}$ ) and residualized hippocampal-FC on residualized memory performance. The significant associations between residualized hippocampal-FC and residualized memory performance found from the prior linear regression results were included. 
To assess the Group $\times$ Hippocampal FC on memory task performance, the average $z$-scored connectivity within the anterior and posterior hippocampi that displayed a significant correlation between ET-related changes in hippocampal FC and memory performance were extracted from subject-level correlation maps of CN participants. Next, ANCOVA was used, with Group and $\triangle$ hippocampal-FC as independent variables, and $\Delta$ memory performance as a dependent variable. The Group $\times \Delta$ hippocampal-FC interaction term was added into the statistical model. Statistical significance was determined at alpha $=0.05$. All statistical tests were conducted using SPSS (v. 26.0, IBM, Armonk, NY).

\section{RESULTS}

\section{Demographic characteristics}

Of the 407 older adults who responded to study recruitment and advertisement, 92 signed the informed consent and underwent neurological examination, 39 individuals started the exercise program, and 35 individuals completed the entire study protocol. Of the 35 participants, 3 individuals (1 MCI and $2 \mathrm{CN}$ ) were excluded due to missing fMRI data. The remaining 32 participants were included in the present analyses (16 MCI and $16 \mathrm{CN}$ ). Among the 16 MCI participants, 6 were amnestic (i.e., impairment in the memory domain). Our past investigation reported complete information about the recruitment and enrollment [22]. Participants had an average age of 77 years and education of 15.8 years. $71 \%$ were women and there were 12 apolipoprotein E epsilon 4 allele (APOE $\varepsilon 4$ ) carriers. The MCI and $\mathrm{CN}$ groups did not significantly differ in the baseline demographic characteristics including age, sex, education, number of $A P O E \varepsilon 4$ carriers, $\dot{\mathrm{VO}}_{2 \text { peak }}$, GDS (missing data $1 \mathrm{MCI}$ and $1 \mathrm{CN}$ ), Mattis Dementia Rating Scale-2 (DRS-2), and Lawton Instrumental Activities of Daily Living (IADL). Table 1 displays demographics, cardiorespiratory fitness, depression, activities of daily living, and cognitive data for all participants.

\section{Aerobic fitness and memory task performance}

$\dot{\mathrm{V}} \mathrm{O}_{2 \text { peak }}$ was significantly increased from before to after ET across participants (missing data $2 \mathrm{CN}$ and $1 \mathrm{MCI})[\mathrm{F}(1,27)=8.632, p=0.007$, $\left.\eta_{p}^{2}=0.242\right]$. There were significant main effects of time, with consistent improvement in the RAVLT
Trial $1\left[\mathrm{~F}(1,30)=5.742, p=0.023, \eta_{\mathrm{p}}^{2}=0.161\right], \mathrm{LM}$ immediate recall $\left[\mathrm{F}(1,30)=9.938, p=0.004, \eta^{2} \mathrm{p}=\right.$ $0.249]$, $\mathrm{LM}$ delayed recall $[\mathrm{F}(1,30)=5.956, p=$ $\left.0.021, \eta_{\mathrm{p}}^{2}=0.166\right]$, and $\mathrm{LM}$ recognition $[\mathrm{F}(1,30)$ $\left.=8.741, p=0.006, \eta_{p}^{2}=0.226\right]$ after ET. Conversely, there were no significant differences between before and after ET in the RAVLT Trial 1-5 [F $\left.(1,30)=2.148, p=0.153, \eta^{2}=0.067\right]$, RAVLT immediate recall $\left[\mathrm{F}(1,30)=0.931, p=0.342, \eta^{2} \mathrm{p}=\right.$ 0.030], RAVLT delayed recall $[\mathrm{F}(1,30)=0.631$, $\left.p=0.433, \eta_{\mathrm{p}}^{2}=0.021\right]$, and RAVLT recognition $\left[\mathrm{F}(1,30)=0.018, \quad p=0.893, \quad \eta^{2} \mathrm{p}=0.001\right] \quad$ (see Table 2).

There were significant main effects of Group on the RAVLT immediate recall $[\mathrm{F}(1,30)=5.201$, $\left.p=0.030, \quad \eta_{\mathrm{p}}^{2}=0.148\right], \quad$ RAVLT delayed recall $\left[\mathrm{F}(1,30)=4.924, p=0.034, \eta_{\mathrm{p}}^{2}=0.141\right]$, LM immediate recall $\left[\mathrm{F}(1,30)=5.270, p=0.029, \eta^{2}{ }_{\mathrm{p}}=0.149\right]$, LM delayed recall $[\mathrm{F}(1,30)=5.070, p=0.032$, $\left.\eta_{\mathrm{p}}^{2}=0.145\right]$, and $\mathrm{LM}$ recognition $[\mathrm{F}(1,30)=7.070$, $\left.p=0.012, \eta_{\mathrm{p}}^{2}=0.191\right]$. These results consistently showed better memory performance in $\mathrm{CN}$ compared to MCI. There were no significant differences between $\mathrm{CN}$ and MCI in the RAVLT Trial 1 $\left[\mathrm{F}(1,30)=0.249, p=0.621, \eta_{\mathrm{p}}^{2}=0.008\right]$, RAVLT Trial 1-5 $\left[\mathrm{F}(1,30)=4.105, p=0.052, \eta_{\mathrm{p}}^{2}=0.120\right]$, and RAVLT recognition $[\mathrm{F}(1,30)=0.079, p=0.780$, $\eta_{\mathrm{p}}^{2}=0.003$ ] (see Table 2).

No significant Group $\times$ Time interactions were found, including the RAVLT Trial 1 $\left[\mathrm{F}(1,30)=1.223, p=0.278, \eta_{\mathrm{p}}^{2}=0.039\right]$, RAVLT Trial 1-5 $\left[\mathrm{F}(1,30)=0.819, p=0.373, \eta_{\mathrm{p}}^{2}=0.027\right]$, RAVLT immediate recall $[\mathrm{F}(1,30)=3.443, p=0.073$, $\left.\eta_{\mathrm{p}}^{2}=0.103\right]$, RAVLT delayed recall $[\mathrm{F}(1,30)=0.463$, $\left.p=0.501, \quad \eta_{\mathrm{p}}^{2}=0.015\right], \quad$ RAVLT recognition $\left[\mathrm{F}(1,30)=0.082, p=0.777, \eta_{\mathrm{p}}^{2}=0.003\right]$, LM immediate recall $\left[\mathrm{F}(1,30)=0.576, p=0.454, \eta_{\mathrm{p}}^{2}=0.019\right]$, LM delayed recall $[\mathrm{F}(1,30)=0.294, p=0.592$, $\left.\eta_{\mathrm{p}}^{2}=0.010\right]$, and LM recognition $[\mathrm{F}(1,30)=0.108$, $\left.p=0.745, \eta_{\mathrm{p}}^{2}=0.004\right]$ (see Table 2).

\section{Voxel-wise hippocampal FC}

We detected no significant changes in hippocampal FC from before to after ET across all participants. Additionally, there were no significant Group $\times$ Time interaction effects. However, when the $\mathrm{CN}$ and MCI groups were analyzed separately, there was a significant increase in FC between the anterior hippocampi and right posterior cingulate (MNI 3 -47 15 [LPI], BA $29,384 \mathrm{~mm}^{3}$ ) in MCI participants after ET compared to before ET (Fig. 2A). No significant ET-induced 


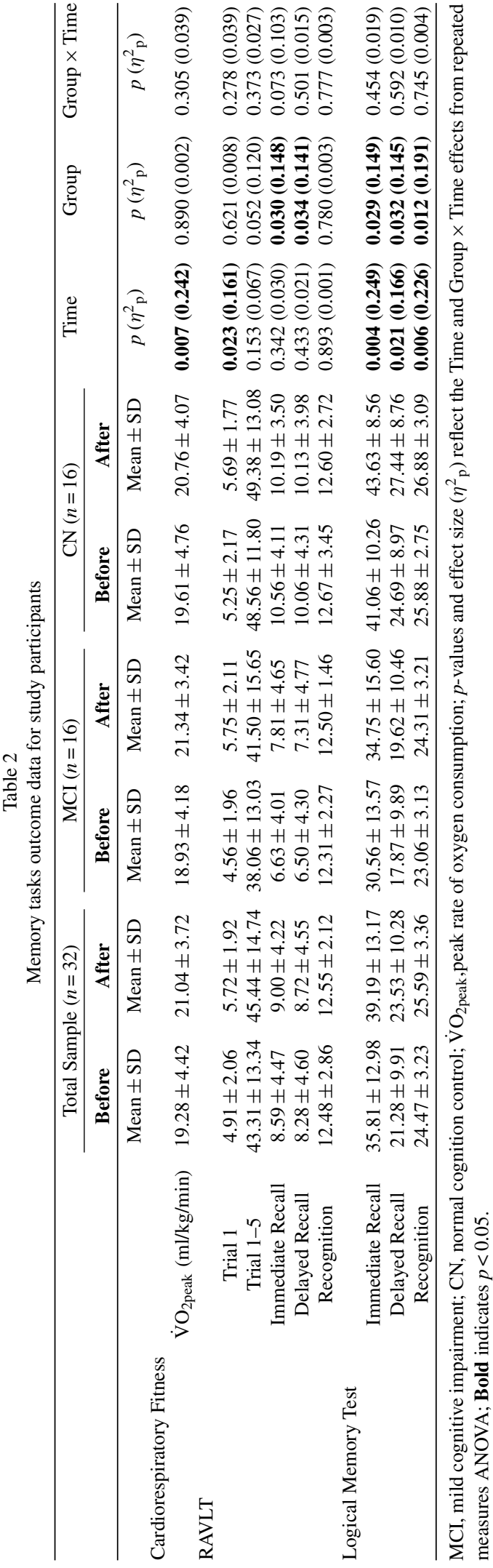

changes in the anterior hippocampi FC were found in $\mathrm{CN}$. There was also increased FC between the posterior hippocampi and regions within the right posterior cingulate (MNI $9-4319$ [LPI], BA 30, $448 \mathrm{~mm}^{3}$ ) (Fig. 2B) in MCI individuals after ET relative to before ET. In $\mathrm{CN}$, increased $\mathrm{FC}$ was detected between the posterior hippocampi and regions within the left cuneus (MNI -11 -75 19 [LPI], BA 18, $384 \mathrm{~mm}^{3}$ ) (Fig. 3A) and left precuneus (MNI -15 -7949 [LPI], BA 7, $336 \mathrm{~mm}^{3}$ ) after ET compared to before ET (Fig. 3B). Lastly, compared to CN, MCI showed significantly lower FC between the posterior hippocampi and left posterior cingulate/precuneus (MNI -7 -51 35 [LPI], $600 \mathrm{~mm}^{3}$ ) at baseline. We observed a similar FC pattern utilizing the bilateral whole hippocampal seed in MCI. In MCI, there was significantly greater FC between whole bilateral hippocampus and bilateral posterior cingulate (MNI 1 -4717 [LPI], BA 30, $648 \mathrm{~mm}^{3}$ ) and right middle temporal gyrus (MNI $51-651$ [LPI], $725 \mathrm{~mm}^{3}$ ). There were no significant bilateral whole hippocampal FC results in CN. Lastly, no significant effects of Time, Group, Group $\times$ Time, and within-group were found in amygdalar FC.

\section{Hippocampal volume}

There were no significant changes in volume of the bilateral hippocampus/TIV across participants from before $(0.0044 \pm 0.0007)$ to after ET $(0.0042$ $\pm 0.0007)\left(p=0.287, \eta_{p}^{2}=0.043\right)$. Also, there were no significant main effect of Group ( $p=0.089$, $\eta_{\mathrm{p}}^{2}=0.107$; MCI before ET: $0.0041 \pm 0.0008$, MCI after ET: $0.0040 \pm 0.0007, \mathrm{CN}$ before ET: $0.00046 \pm 0.0005, \mathrm{CN}$ after ET: $0.0044 \pm 0.0006)$ or Group $\times$ Time effects $\left(p=0.439, \eta^{2}{ }_{\mathrm{p}}=0.023\right)$. We also found no significant associations between the $\Delta$ hippocampal FC (significant results shown in Figs. 2 and 3 ) and baseline (all $p$-values $\geq 0.188$ ) and ET-induced changes (all $p$-values $\geq 0.143$ ) in hippocampal volume in both MCI and CN. As there were no ET-related changes, hippocampal volume was not included as a covariate when we assessed the association between $\Delta$ hippocampal volume and $\Delta$ memory performance.

\section{Associations between changes in hippocampal $F C$ and memory performance}

In MCI, the $\Delta$ anterior hippocampus-right posterior cingulate connectivity explained $38.3 \%$ of $\Delta \mathrm{LM}$ recognition $\left[R=0.619, R^{2}=0.383, p=0.014,95 \%\right.$ 
A

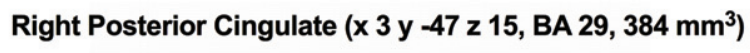
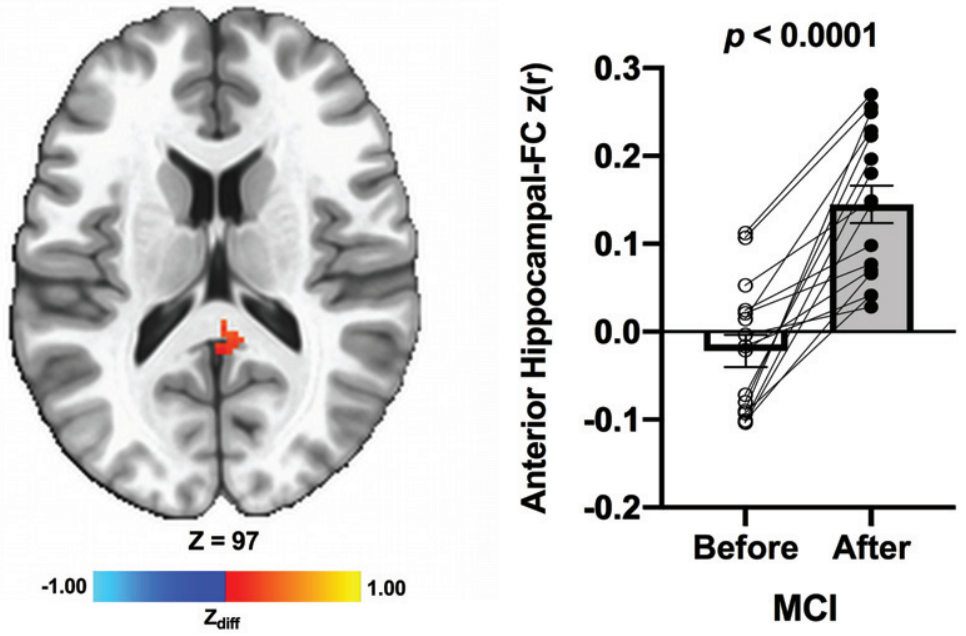

B

\section{Right Posterior Cingulate (x 9 y -43 z 19, BA 30, $448 \mathrm{~mm}^{3}$ )}
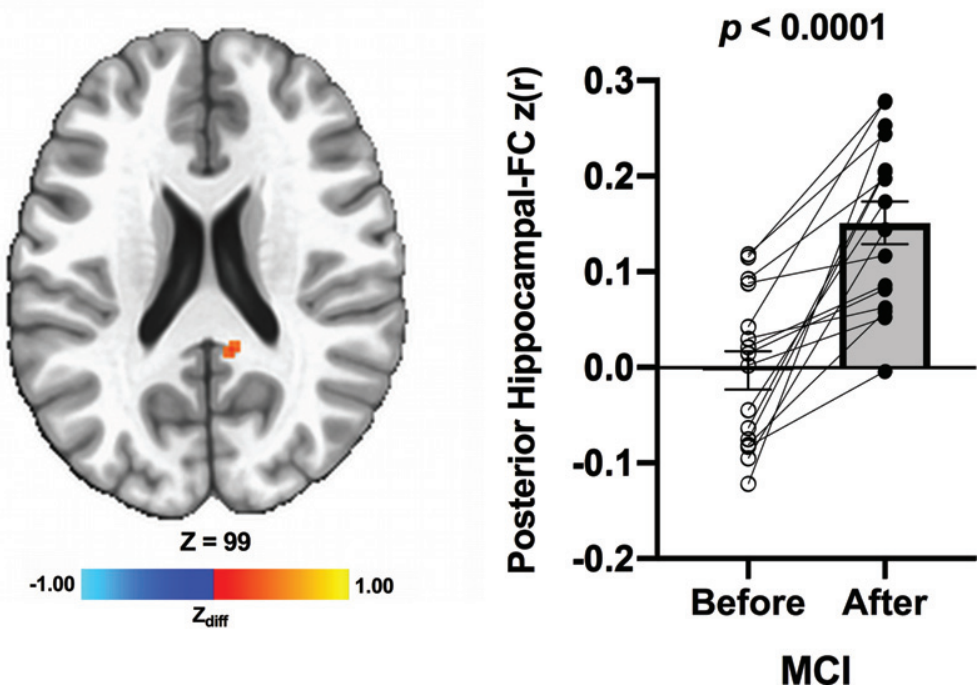

Fig. 2. Increased functional connectivity between (A) the anterior hippocampal seed and (B) the posterior hippocampal seed and region within the right posterior cingulate, respectively, were found from before to after ET in individuals with MCI. Adjacent bar graphs indicate the connectivity between each hippocampal seed and right posterior cingulate ( $\pm \mathrm{SEM}$ ) for before and after ET. $p$-values above bar graphs indicate statistical difference from before to after ET.

CI 3.844, 28.330] (Fig. 4A) and the $\Delta$ posterior hippocampus-right posterior cingulate connectivity explained $37.6 \%$ of $\Delta \mathrm{LM}$ recognition $[R=0.613$, $R^{2}=0.376, \quad p=0.015,95 \%$ CI 3.344, 25.971] (Fig. 4B). Furthermore, the $\Delta$ bilateral whole hippocampus-bilateral posterior cingulate connectivity
(Supplementary Figure 1A) explained $27.4 \%$ of $\Delta \mathrm{LM}$ recognition $\left[R=0.650, R^{2}=0.422, p=0.009\right.$, 95\% CI 4.676, 26.597] (Supplementary Figure 1B) in MCI. Conversely, in $\mathrm{CN}$, neither $\Delta$ posterior hippocampus-left cuneus connectivity $[R=0.091$, $R^{2}=0.008, p=0.748,95 \%$ CI $\left.-19.807,14.591\right]$, nor 
$\triangle$ posterior hippocampus-left precuneus connectivity $\left[R=0.051, R^{2}=0.003, p=0.857,95 \%\right.$ CI -17.389 , 20.614] were associated with $\Delta \mathrm{LM}$ recognition. In addition, none of the significant association between $\Delta$ posterior hippocampal-FC and other memory task performance was found in both $\mathrm{MCI}$ and $\mathrm{CN}$ $(p \geq 0.214)$.

\section{Group (MCI versus $C N$ ) interaction on the association between hippocampal FC and LM recognition}

While there was no significant interaction between Group and $\Delta$ anterior hippocampal-FC (with right posterior cingulate) on $\triangle \mathrm{LM}$ recognition $\left[\mathrm{F}(1,28)=1.842, p=0.186, \eta_{\mathrm{p}}^{2}=0.068\right]$, the interaction between $\Delta$ posterior hippocampal-FC (with right posterior cingulate) on $\triangle \mathrm{LM}$ recognition $\left[\mathrm{F}(1,28)=3.438, p=0.075, \eta^{2} \mathrm{p}=0.116\right]$ approached the borderline of significance.

\section{DISCUSSION}

The present study examined the effects of a 12-week walking ET intervention on hippocampal FC and its association with learning and recognition memory task performance in $\mathrm{CN}$ older adults and older adults diagnosed with MCI. The walking exercise intervention evoked a $10.5 \%$ increase in cardiorespiratory fitness $\left(\dot{\mathrm{VO}}_{2 \text { peak }}\right)$ across participants. Consistent with our hypotheses, a 12-week ET intervention led to increased hippocampal FC in older adults. Notably, there were no correlations between ET-related changes in hippocampal FC and baseline hippocampal volume or ET-related changes in hippocampal volume. Thus, the changes in hippocampal FC occurred independent of possible (yet undetectable) changes in hippocampal volume, suggesting that these effects of ET were specific to the functional connections and extended network of the hippocampus. Furthermore, the lack of significant changes in amygdalar FC in response to ET suggests that hippocampal networks may be particularly sensitive to the effects of chronic exercise. While increased hippocampal FC following ET was specific to the posterior hippocampal seeds in cognitively asymptomatic individuals, both anterior and posterior hippocampal FC were enhanced in MCI participants. In MCI, the ET-induced increase in hippocampal FC was associated with significantly enhanced recognition during the LM test. In contrast, there were no significant relationships between ET-elicited changes in hippocampal FC and hippocampal-dependent task performance in $\mathrm{CN}$ older adults.

The ET-induced increase in hippocampal FC we observed in older adults contrasts with a prior finding that reported no significant changes in hippocampal FC following 6 months of aerobic exercise compared to a stretching and toning control group in healthy older adults. However, in their post-hoc analysis using a BOLD signal fluctuations approach, there was an association between ET-induced improvements in cardiorespiratory fitness and decreased hippocampal FC across participants [38]. Conversely, there was no significant relationship between ET-related changes in $\dot{\mathrm{V}}_{2 \text { peak }}$ and hippocampal FC $(\mathrm{CN}, p=0.435$; MCI, $p=0.392$; data not presented) in the present study. Differences in the directionality of hippocampal FC between Flodin et al. (2017) [38] and the current study might arise from discrepancies in the analytic approach (BOLD time series fluctuation versus whole-brain), study design (intervention duration; exercise mode), and participant characteristics (without versus with MCI participants). Although it may suggest a shift in attentional allocation, negative FC between brain regions is typically difficult to interpret [20], particularly when these changes were not related to behavior. Flodin et al. (2017) [38] lacked associations between connectivity and cognitive performance, which clouds the interpretation of the functional meaning of their fitness-related associations with changes in hippocampal FC, and so direct comparison to the present results is difficult.

Regarding connectivity-cognition associations, our results are consistent with those of a prior study that assessed the effects of a 12-week Tai Chi Chuan and Badunjin practice on the hippocampal functional network and its association with memory function using Wechsler Memory Scale-Chinese Version in older adults (ages 50-70) [19]. Results showed that Tai Chi practice significantly enhanced FC between bilateral hippocampus and medial prefrontal gyrus, which was significantly associated with a corresponding improvement in memory. Key differences between Tao et al. (2016) [39] and the present study include exercise mode (mind-body versus aerobic exercise), selection of hippocampal seeds (whole versus anterior/posterior hippocampus), and cognitive status of the sample (without versus with MCI individuals). These differences in study design and analytic methods may be responsible for disparities in the brain regions showing increased FC with hippocampus. Despite these differences, both studies are consistent in the finding that ET increased 
A

Left Cuneus (x -11 y -75 z 19, BA 18, $384 \mathrm{~mm}^{3}$ )
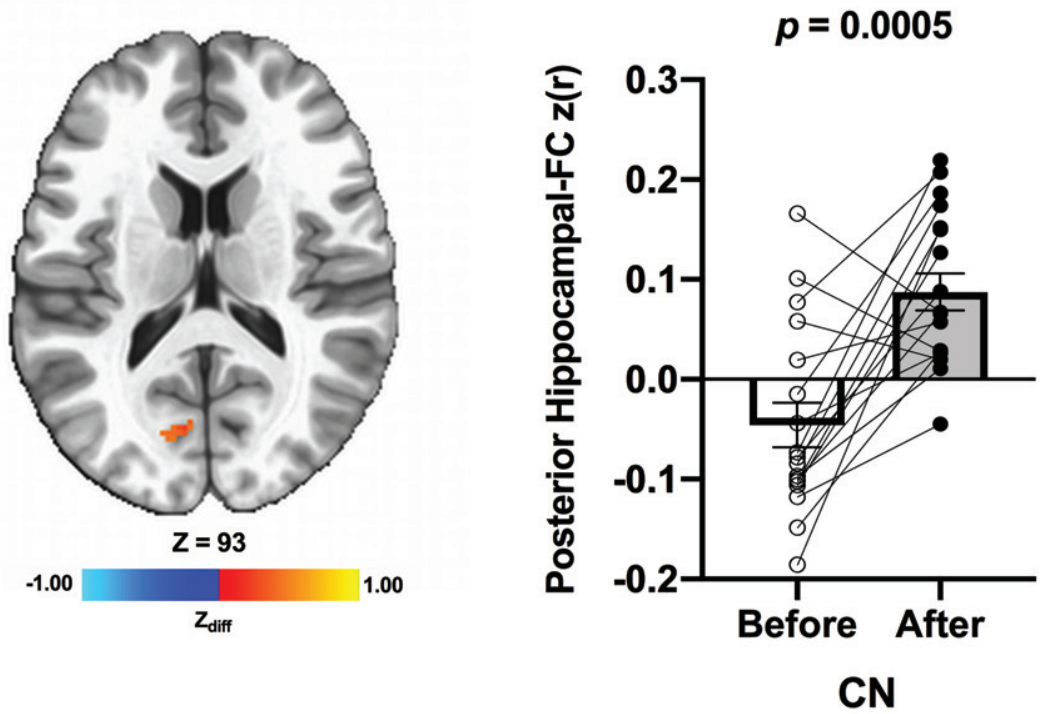

B

Left Precuneus ( $x-15$ y -79 z 49, BA 7, $336 \mathrm{~mm}^{3}$ )

$$
p=0.0001
$$
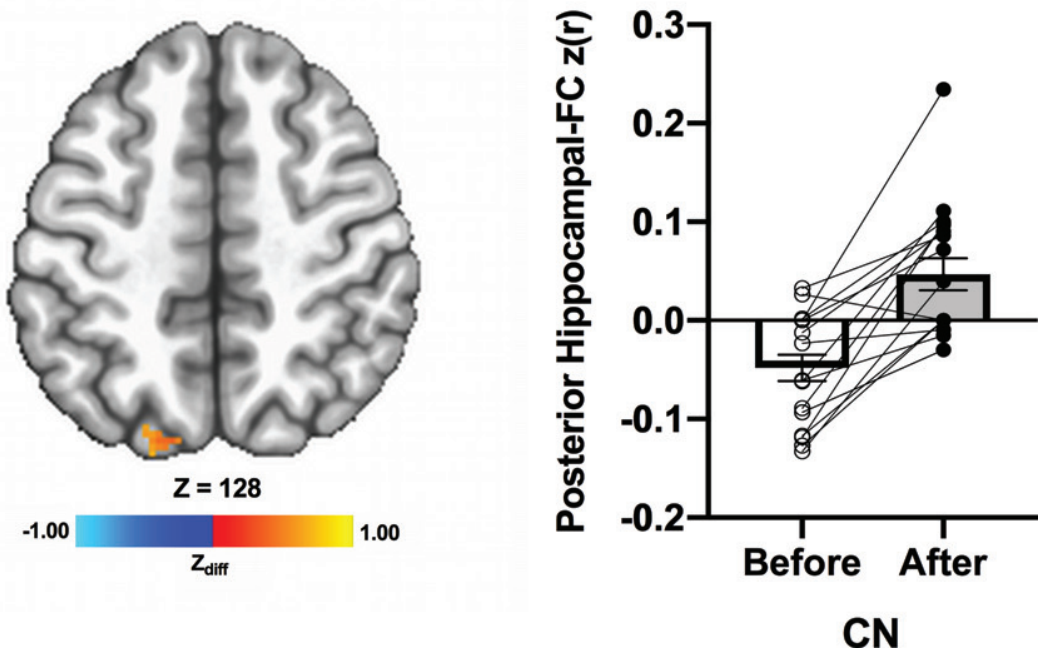

Fig. 3. Increased functional connectivity between posterior hippocampal seed and (A) left cuneus and (B) left precuneus, respectively, were found from before to after ET in CN individuals. Adjacent bar graphs indicate the connectivity between each hippocampal seed and right posterior cingulate $( \pm \mathrm{SEM})$ for before and after ET. $p$-values above bar graphs indicate statistical difference from before to after ET.

hippocampal FC, which was also related to enhanced memory performance in older adults.

It is notable that there were no significant hippocampal volume changes in the MCI individuals, which is consistent with the exercise training literature in MCI. It is also plausible to hypothesize that increased hippocampal connectivity may cooccur with other protective effects associated with maintenance of the structural integrity in the hippocampus among cognitively healthy older adults and those diagnosed with MCI. Nonetheless, the significant alterations in hippocampal FC observed following 12 weeks of ET implies that functional changes in the hippocampus, and with its extended functional network, may occur in the absence of measurable volumetric alterations. The lack of 
association between baseline hippocampal volume and change in hippocampal connectivity in both MCI and $\mathrm{CN}$ groups further suggests that ET-related plasticity in hippocampal functional networks may occur independently of disease-related atrophy. However, these hypotheses are speculative due to our small sample size, relatively short intervention period, and lack of measures of brain amyloid and tau. Hence, future studies need to robustly test this hypothesis by employing a large sample size, a longer intervention period (e.g., 6 months or 12 months), and including estimates of $\mathrm{AD}$ pathology.

Prior human investigations suggested that the salutary effects on hippocampal volume elicited by greater cardiorespiratory fitness appeared to be specific to the anterior relative to the posterior region [13, 40]. Using a functional connectivity approach in the present study, we found that both anterior and posterior hippocampal FC were increased in MCI, and only markedly enhanced posterior hippocampal FC was found in the $\mathrm{CN}$ group. This extends previous exercise neuroimaging studies by suggesting that ET-induced effects on the hippocampus may not be confined to the anterior region, but posterior hippocampus may also play an important role in ET-modulated improvement in episodic memory performance. There is evidence supporting the connection between the posterior hippocampus and the default mode network (DMN) that included anterior/posterior cingulate and precuneus, and its close relevance to episodic memory retrieval [41]. In support, our previous investigation using the same sample showed increased connectivity between the DMN (the posterior cingulate/precuneus as a seed region) and right parahippocampal gyrus after the 12-week walking intervention compared to baseline, whereas connectivity with the DMN was not altered in the $\mathrm{CN}$ participants [21]. In line with these prior works, the brain regions showing increased FC with hippocampus in the current study were centered on primary hub regions of the DMN (e.g., posterior cingulate and precuneus). These associations between recognition memory performance improvements and increased connectivity between the posterior cingulate/precuneus and both anterior and posterior hippocampal subregions in the MCI participants further substantiate recent perspectives regarding the roles of anterior and posterior hippocampal connections to memory networks $[42,43]$.

In the present study, the increased FC between anterior/posterior hippocampus and right posterior cingulate was related to improved LM recognition in MCI. Performing the LM test requires memoryencoding, storage, and recall procedure and the test is considered as an effective measurement to assess episodic memory [44]. The LM test is also sensitive to detect cognitive decline [45], is able to identify subtle alterations in memory, and predicts conversion to $\mathrm{AD}$ in MCI [46]. Enhanced LM performances after exercise intervention in this study indicates that ET not only delays the expected cognitive decline, but also results in cognitive improvements in individuals diagnosed with MCI. Although speculative, the fact that the significant associations between hippocampal FC and recognition memory performance were observed in MCI implicates ET-induced increases in hippocampal FC as an underlying neurophysiological mechanism that may drive ET-related enhancement in memory among older individuals with MCI, presumed to be on the AD continuum. Conversely, there were no associations between the hippocampal $\mathrm{FC}$ and learning and recognition memory performance in the $\mathrm{CN}$ group, indicating that cognitively intact controls may have different neurophysiological mechanism in the ET-related memory improvements compared to their MCI counterparts.

Group differences (MCI versus $\mathrm{CN}$ ) were shown both in hippocampal FC and memory performance in our study. As expected, the $\mathrm{CN}$ group consistently outperformed the MCI group in neuropsychological testing, including recall and recognition memory performance (e.g., RAVLT immediate and delayed recall, and LM immediate recall, delayed recall, and recognition). At baseline, there was also significantly lower FC between posterior hippocampus and left posterior cingulate/precuneus in MCI compared to $\mathrm{CN}$. Our results concur with previous evidence demonstrating poorer memory performance [47] and disrupted hippocampal FC [48] in MCI compared to $\mathrm{CN}$ older adults. Yet, despite the baseline disparities in hippocampal function and memory performance between $\mathrm{CN}$ and MCI, ET was equally beneficial in both groups' hippocampal FC and hippocampal-dependent task performance. Viewed from this perspective, regular participation in walking exercise may counteract age- and AD-related hippocampal deficits, even among older adults who have experienced cognitive decline. Importantly, the ET-related brain health improvements in individuals with MCI are corroborated by our prior investigations (using the same sample analyzed in the present study) that demonstrated an increased DMN [21] and cerebellar FC [49], enhanced neural efficiency during a semantic memory task [22], positive associations 
between changes in fitness and changes in cortical thickness [50], and reduced cerebral blood flow that corresponded to improvement in phonemic fluency task performance [51] after ET.

\section{Potential mechanisms}

ET is associated with enhancing mitochondrial function in the hippocampal regions, which is instrumental for synaptogenesis (i.e., growth of new synapses) and dendritic complexity [52]. Chronic exercise therefore promotes synaptic strength or long-term potentiation (LTP), one of the key cellar mechanisms that underlies learning and memory [53] and the effects of ET on synaptic strengthening is particularly evident in the dentate gyrus, a hippocampal subregion [54]. These ET-related adaptations seem to facilitate construction of a stronger neural scaffolding [55], engendering resilient capacity within the neural networks to withstand age- or pathology-related brain dysfunction [56-59]. A common characteristic of normal aging and $\mathrm{AD}$ pathology progression is an adaptive compensatory response through increased neural activation (i.e., recruitment of additional neural resources) [55]. While the compensatory response may be a considered harbinger of eventual cognitive decline, it is also indicative of a dynamic adaption to maintain function in the face of both normal aging and AD-related pathology [21]. Indeed, aging brains that exhibited more extended compensatory responses maintained cognitive function for a longer period $[60,61]$.

Based on these neurophysiological mechanisms, we surmise that ET-related hippocampal adaptations, if they indeed occur in individuals diagnosed with MCI, were exhibited through greater connectivity of the hippocampus, a brain region preferentially targeted in AD-related neurodegenerative processes [62]. Corroborating this hypothesis, our results revealed that increased connectivity following ET appeared primarily between the hippocampus and the DMN whose key regions include posterior cingulate and precuneus [63]. Given that both hippocampus and DMN are susceptible to AD pathology (e.g., processes associated with amyloid accumulation and/or retention) [64] and have been reported to manifest compensatory brain activation in response to AD-related neural pathology [21, 61], the greater connectivity observed between the hippocampus and posterior cingulate/precuneus in the present study is likely to reflect beneficial compensatory responses induced by ET.
Moreover, the pattern of stronger hippocampal connectivity and improved hippocampal-dependent task performance shown in MCI individuals after ET is a marked contrast to MCI patients who typically experience AD-related loss of hippocampal connectivity [65] and progressive memory impairment [47]. It may be that regular participation in exercise plays a protective role in reversing, or stabilizing, the expected trend of disrupted connectivity and memory loss in MCI. Thus, the increased hippocampal compensatory response in both $\mathrm{MCI}$ and $\mathrm{CN}$ older adults after a 12-week walking intervention may delineate maintained or improved neuronal and cognitive functions, which were also evidenced by enhanced memory performance after ET. However, this hypothesis should be further explored as we did not measure other AD biomarkers, such as beta-amyloid load or other indices of neurological damage, such as phosphorylated tau.

\section{Strengths and limitations}

One of the major strengths of the present study was the well-attended (compliance rate of $\sim 96 \%$ ) and well-supervised intervention, which contributed to successfully increasing cardiorespiratory fitness in response to ET ( $10.5 \%$ increase in $\left.\dot{\mathrm{VO}}_{2 \text { peak }}\right)$. Next, older adults with an objective diagnosis of MCI, who have been understudied in the aging and exercise neuroscience literature, were included. Additionally, to preclude the vulnerability to noise and likelihood of cluster-level false positive rate, we used a stringent voxel-wise probability threshold $(p<0.001)$ in compliance with the current fMRI thresholding recommendations [66].

Despite these strengths, the present study is subject to the limitation of lacking a non-exercise (or active) control group, warranting some caution in interpreting the results until they can be replicated in a larger randomized controlled trial. Nevertheless, our findings are consistent with additional effects we have observed in this study sample [51] and our results are in line with well-documented effects of ET on the hippocampus [67]. Because LM does not have alternate forms, some improvement due to a practice effect might be expected. Nevertheless, we used residualized change scores, which adjusts for influences of baseline performance, practice effects and regression to the mean $[37,68$, 69]. Thus, the cognitive improvement in our sample may exceed what could be expected with repeated test administration. Furthermore, group differences 
A

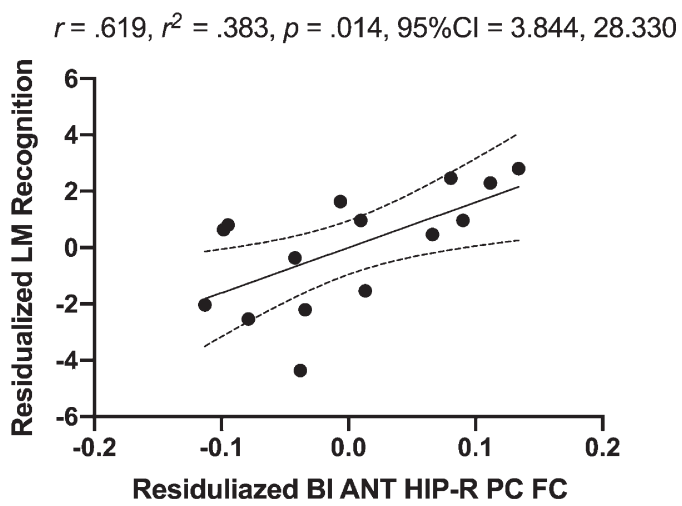

B

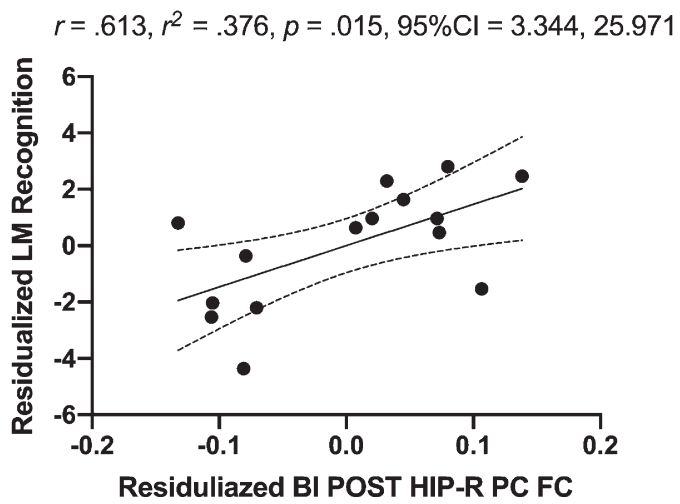

Fig. 4. Positive associations of residualized changes in anterior hippocampal-FC (anterior hippocampus and right posterior cingulate; Fig. 2A) and residualized changes in logical memory recognition performance in MCI individuals (A). There was also a positive correlation between of residualized changes in posterior hippocampal-FC (posterior hippocampus and right posterior cingulate; Fig. 2B) and residualized changes in logical memory recognition performance in MCI individuals (B). Dotted curves indicate confidence interval around the mean. CI, 95\% confidence interval; BI, bilateral; ANT, anterior; POST, posterior; HIP, hippocampal; R, right; $\mathrm{PC}$, posterior cingulate.

would not be expected due to practice effects because both groups experienced the same task conditions. Indeed, improvement in memory performance after ET among individual diagnosed with MCI reflects a meaningful change. Achieving cognitive stability in older adults diagnosed with MCI is more often a sign of treatment success in exercise [70], cognitive training [71], and pharmacologic interventions [72]. Furthermore, the improvements in memory performance in MCI corresponded to the changes in hippocampal FC, adding support for the hypothesis that ET may impact memory-related neural networks. Of note, the consistency of the magnitude and direction of the cognition-connectivity association in the MCI group gives some confidence that these findings were not merely spurious. Hence, our results are not likely to simply reflect passage of time or other nonspecific intervention effects. The present study is also limited by a relatively small sample size $(n=32)$ and homogeneous characteristics of participants (e.g., most of the participants were women (71\%) and highly educated (15.8 years of education)) and so may not be generalizable to the entire older adult population. Lastly, using a 12 -week intervention, we may have missed potential effects that could manifest after a longer exercise intervention (e.g., 6-12 months). Yet, our data suggest ET-related effects on hippocampal connectivity and cognition may emerge within three months, and thus it may be useful for longer interventions to sample more than just before and after the intervention in order to document the time course of these effects.

\section{CONCLUSION}

In summary, 12 weeks of regular walking ET improved the RAVLT and LM test performance in older adults with both intact and impaired cognition. The FC increase between posterior hippocampus and brain regions in the DMN may be an important determinant of the ET-induced enhancements in recognition memory among older adults diagnosed with MCI. ET-related adaptations may have induced increased neural synchronization in the hippocampal network and led to the creation of a stronger neural scaffolding and a greater compensatory response. The ET-related increase in hippocampal FC occurred independent of initial hippocampal volume or changes in hippocampal volume, suggesting that effects induced by a 12-week walking ET were specific to the functional network of the hippocampus. In addition, as there were no significant changes in amygdalar FC, the hippocampus was the region preferentially and specifically affected by chronic exercise in the medial temporal lobe. The current findings also extend the existing literature by elucidating that ET-related salutary effects on the hippocampus may not be confined to the anterior region, as connectivity with the posterior hippocampus was associated with an enhancement in ET-related episodic memory performance. As a final point, the older adults diagnosed with MCI who participated in this study remained cognitively impaired relative to the cognitively normal participants at the conclusion of the 
study. Even so, these findings provide additional evidence that a lifestyle intervention as simple as moderate-intensity walking may generate neural network plasticity that impacts memory performance in older adults facing a diagnosis of MCI due to AD.

\section{ACKNOWLEDGMENTS}

We thank the participants for their dedication while participating in this study, Drs. Nathan Hantke and Alissa Butts for their assistance with participant assessment, and Dr. Piero Antuono for his role in diagnosis of MCI. This study was supported by the University of Wisconsin-Milwaukee Graduate School Research Growth Initiative; and the National Center for Advancing Translational Sciences, NIH grant numbers 8UL1TR000055, 8KL2TR000056. The content is solely the responsibility of the authors and does not necessarily represent the official views of the NIH.

Authors' disclosures available online (https:// www.j-alz.com/manuscript-disclosures/21-0051r2).

\section{SUPPLEMENTARY MATERIAL}

The supplementary material is available in the electronic version of this article: https://dx.doi.org/ 10.3233/JAD-210051.

\section{REFERENCES}

[1] Grady CL, Craik FI (2000) Changes in memory processing with age. Curr Opin Neurobiol 10, 224-231.

[2] Small SA, Schobel SA, Buxton RB, Witter MP, Barnes CA (2011) A pathophysiological framework of hippocampal dysfunction in ageing and disease. Nat Rev Neurosci 12, 585-601.

[3] Mega MS, Small GW, Xu ML, Felix J, Manese M, Tran NP, Dailey JI, Ercoli LM, Bookheimer SY, Toga AW (2002) Hippocampal atrophy in persons with age-associated memory impairment: Volumetry within a common space. Psychosom Med 64, 487-492.

[4] Heo S, Prakash RS, Voss MW, Erickson KI, Ouyang C, Sutton BP, Kramer AF (2010) Resting hippocampal blood flow, spatial memory and aging. Brain Res 1315, 119-127.

[5] Rosenzweig ES, Barnes CA (2003) Impact of aging on hippocampal function: Plasticity, network dynamics, and cognition. Prog Neurobiol 69, 143-179.

[6] Navarro A, López-Cepero JM, Bández MJ, Sánchez-Pino M-J, Gómez C, Cadenas E, Boveris A (2008) Hippocampal mitochondrial dysfunction in rat aging. Am J Physiol-Regul Integr Comp Physiol 294, R501-R509.

[7] Petersen RC (2000) Mild cognitive impairment: Transition between aging and Alzheimer's disease. Neurologia 15, 93-101.

[8] Hanseeuw BJ, Schultz AP, Betensky RA, Sperling RA, Johnson KA (2016) Decreased hippocampal metabolism in high-amyloid mild cognitive impairment. Alzheimers Dement 12, 1288-1296.

[9] Broadhouse KM, Mowszowski L, Duffy S, Leung I, Cross N, Valenzuela MJ, Naismith SL (2019) Memory performance correlates of hippocampal subfield volume in mild cognitive impairment subtype. Front Behav Neurosci 13, 259.

[10] Family Caregiver Alliance. Incidence and prevalence of the major causes of brain impairment. https://www. caregiver.org/resource/incidence-and-prevalence-majorcauses-brain-impairment/

[11] Boyle PA, Wilson RS, Aggarwal NT, Tang Y, Bennett DA (2006) Mild cognitive impairment: Risk of Alzheimer disease and rate of cognitive decline. Neurology 67, 441-445.

[12] Hillman CH, Erickson KI, Kramer AF (2008) Be smart, exercise your heart: Exercise effects on brain and cognition. Nat Rev Neurosci 9, 58-65.

[13] Erickson KI, Voss MW, Prakash RS, Basak C, Szabo A, Chaddock L, Kim JS, Heo S, Alves H, White SM (2011) Exercise training increases size of hippocampus and improves memory. Proc Natl Acad Sci U S A 108, 3017-3022.

[14] van Praag H, Shubert T, Zhao C, Gage FH (2005) Exercise enhances learning and hippocampal neurogenesis in aged mice. J Neurosci 25, 8680-8685.

[15] Nagamatsu LS, Chan A, Davis JC, Beattie BL, Graf P, Voss MW, Sharma D, Liu-Ambrose T (2013) Physical activity improves verbal and spatial memory in older adults with probable mild cognitive impairment: A 6-month randomized controlled trial. J Aging Res 2013, 861893.

[16] Ten Brinke LF, Bolandzadeh N, Nagamatsu LS, Hsu CL, Davis JC, Miran-Khan K, Liu-Ambrose T (2015) Aerobic exercise increases hippocampal volume in older women with probable mild cognitive impairment: A 6month randomised controlled trial. Br J Sports Med 49, 248-254.

[17] Fox MD, Snyder AZ, Vincent JL, Corbetta M, Van Essen DC, Raichle ME (2005) The human brain is intrinsically organized into dynamic, anticorrelated functional networks. Proc Natl Acad Sci U S A 102, 9673-9678.

[18] Damoiseaux JS, Rombouts S, Barkhof F, Scheltens P, Stam CJ, Smith SM, Beckmann CF (2006) Consistent restingstate networks across healthy subjects. Proc Natl Acad Sci U S A 103, 13848-13853.

[19] Tao J, Liu J, Egorova N, Chen X, Sun S, Xue X, Huang J, Zheng G, Wang Q, Chen L (2016) Increased hippocampus-medial prefrontal cortex resting-state functional connectivity and memory function after Tai Chi Chuan practice in elder adults. Front Aging Neurosci 8, 25.

[20] Stillman CM, Uyar F, Huang H, Grove GA, Watt JC, Wollam ME, Erickson KI (2018) Cardiorespiratory fitness is associated with enhanced hippocampal functional connectivity in healthy young adults. Hippocampus 28, 239-247.

[21] Chirles TJ, Reiter K, Weiss LR, Alfini AJ, Nielson KA, Smith JC (2017) Exercise training and functional connectivity changes in mild cognitive impairment and healthy elders. J Alzheimers Dis 57, 845-856.

[22] Smith JC, Nielson KA, Antuono P, Lyons J-A, Hanson RJ, Butts AM, Hantke NC, Verber MD (2013) Semantic memory functional MRI and cognitive function after exercise intervention in mild cognitive impairment. J Alzheimers Dis 37, 197-215.

[23] Oldfield RC (1971) The assessment and analysis of handedness: The Edinburgh inventory. Neuropsychologia 9, 97-113. 
[24] Yesavage JA (1988) Geriatric depression scale. Psychopharmacol Bull 24, 709-711.

[25] Lawton MP, Brody EM (1969) Assessment of older people: Self-maintaining and instrumental activities of daily living. Gerontologist 9, 179-186.

[26] Albert MS, DeKosky ST, Dickson D, Dubois B, Feldman HH, Fox NC, Gamst A, Holtzman DM, Jagust WJ, Petersen RC (2011) The diagnosis of mild cognitive impairment due to Alzheimer's disease: Recommendations from the National Institute on Aging-Alzheimer's Association workgroups on diagnostic guidelines for Alzheimer's disease. Alzheimers Dement 7, 270-279.

[27] Rey A (1958) L'examen clinique en psychologie.

[28] Sullivan K (2005) Alternate forms of prose passages for the assessment of auditory-verbal memory. Arch Clin Neuropsychol 20, 745-753.

[29] Medicine AC of S (2013) ACSM's guidelines for exercise testing and prescription, Lippincott Williams \& Wilkins.

[30] Borg G (1998) Borg's perceived exertion and pain scales. Human kinetics.

[31] Fischl B (2012) FreeSurfer. Neuroimage 62, 774-781.

[32] Cox RW (1996) AFNI: Software for Analysis and Visualization of Functional Magnetic Resonance Neuroimages. Comput Biomed Res 29, 162-173.

[33] Beall EB, Lowe MJ (2014) SimPACE: Generating simulated motion corrupted BOLD data with syntheticnavigated acquisition for the development and evaluation of SLOMOCO: A new, highly effective slicewise motion correction. Neuroimage 101, 21-34.

[34] Raz N, Lindenberger U, Rodrigue KM, Kennedy KM, Head D, Williamson A, Dahle C, Gerstorf D, Acker JD (2005) Regional brain changes in aging healthy adults: General trends, individual differences and modifiers. Cereb Cortex 15, 1676-1689.

[35] Won J, Alfini AJ, Weiss LR, Nyhuis CC, Spira AP, Callow DD, Carson Smith J (2019) Caudate volume mediates the interaction between total sleep time and executive function after acute exercise in healthy older adults. Brain Plast $\mathbf{5}$, 69-82.

[36] Llabre MM, Spitzer SB, Saab PG, Ironson GH, Schneiderman N (1991) The reliability and specificity of delta versus residualized change as measures of cardiovascular reactivity to behavioral challenges. Psychophysiology $\mathbf{2 8}$, 701-711.

[37] Temkin NR, Heaton RK, Grant I, Dikmen SS (1999) Detecting significant change in neuropsychological test performance: A comparison of four models. J Int Neuropsychol Soc 5, 357-369.

[38] Flodin P, Jonasson LS, Riklund K, Nyberg L, Boraxbekk C-J (2017) Does aerobic exercise influence intrinsic brain activity? An aerobic exercise intervention among healthy old adults. Front Aging Neurosci 9, 267.

[39] Gong Y, Wang DJ, Deng J (1989) Handbook of Wechsler memory scale-revised. Bulletin of Human Medical College, Changsha.

[40] Thomas AG, Dennis A, Rawlings NB, Stagg CJ, Matthews L, Morris M, Kolind SH, Foxley S, Jenkinson M, Nichols TE (2016) Multi-modal characterization of rapid anterior hippocampal volume increase associated with aerobic exercise. Neuroimage 131, 162-170.

[41] Poppenk J, Moscovitch M (2011) A hippocampal marker of recollection memory ability among healthy young adults: Contributions of posterior and anterior segments. Neuron 72, 931-937.
[42] Sekeres MJ, Winocur G, Moscovitch M (2018) The hippocampus and related neocortical structures in memory transformation. Neurosci Lett 680, 39-53.

[43] Sheldon S, Levine B (2016) The role of the hippocampus in memory and mental construction. Ann N Y Acad Sci 1369, 76-92.

[44] Li M, Ng TP, Kua EH, Ko SM (2006) Brief informant screening test for mild cognitive impairment and early Alzheimer's disease. Dement Geriatr Cogn Disord 21, 392-402.

[45] Dunn JC, Almeida OP, Barclay L, Waterreus A, Flicker L (2002) Latent semantic analysis: A new method to measure prose recall. J Clin Exp Neuropsychol 24, 26-35.

[46] Robinson-Whelen S, Storandt M (1992) Immediate and delayed prose recall among normal and demented adults. Arch Neurol 49, 32-34.

[47] Greenaway MC, Lacritz LH, Binegar D, Weiner MF, Lipton A, Cullum CM (2006) Patterns of verbal memory performance in mild cognitive impairment, Alzheimer disease, and normal aging. Cogn Behav Neurol 19, 79-84.

[48] Zhou Y, Dougherty Jr JH, Hubner KF, Bai B, Cannon RL, Hutson RK (2008) Abnormal connectivity in the posterior cingulate and hippocampus in early Alzheimer's disease and mild cognitive impairment. Alzheimers Dement 4, 265-270.

[49] Won J, Faroqi-Shah Y, Callow DD, Williams A, Awoyemi A, Nielson KA, Smith JC (2021) Association between greater cerebellar network connectivity and improved phonemic fluency performance after exercise training in older adults. Cerebellum, doi: 10.1007/s12311-020-01218-3.

[50] Reiter K, Nielson KA, Smith TJ, Weiss LR, Alfini AJ, Smith JC (2015) Improved cardiorespiratory fitness is associated with increased cortical thickness in mild cognitive impairment. J Int Neuropsychol Soc 21, 757-767.

[51] Alfini AJ, Weiss LR, Nielson KA, Verber MD, Smith JC (2019) Resting cerebral blood flow after exercise training in mild cognitive impairment. J Alzheimers Dis 67, 671-684.

[52] Steib K, Schäffner I, Jagasia R, Ebert B, Lie DC (2014) Mitochondria modify exercise-induced development of stem cell-derived neurons in the adult brain. J Neurosci 34, 6624-6633.

[53] Bettio L, Thacker JS, Hutton C, Christie BR (2019) Modulation of synaptic plasticity by exercise. Int Rev Neurobiol 147, 295-322.

[54] Patten AR, Sickmann H, Hryciw BN, Kucharsky T, Parton R, Kernick A, Christie BR (2013) Long-term exercise is needed to enhance synaptic plasticity in the hippocampus. Learn Mem 20, 642-647.

[55] Reuter-Lorenz PA, Park DC (2014) How does it STAC up? Revisiting the scaffolding theory of aging and cognition. Neuropsychol Rev 24, 355-370.

[56] Hyodo K, Dan I, Suwabe K, Kyutoku Y, Yamada Y, Akahori M, Byun K, Kato M, Soya H (2012) Acute moderate exercise enhances compensatory brain activation in older adults. Neurobiol Aging 33, 2621-2632.

[57] Meeusen R, Smolders I, Sarre S, De Meirleir K, Keizer H, Serneels M, Ebinger G, Michotte Y (1997) Endurance training effects on neurotransmitter release in rat striatum: An in vivo microdialysis study. Acta Physiol Scand 159, 335-341.

[58] Won J, Alfini AJ, Weiss LR, Michelson CS, Callow DD, Ranadive SM, Gentili RJ, Smith JC (2019) Semantic memory activation after acute exercise in healthy older adults. $J$ Int Neuropsychol Soc 25, 557-568. 
[59] Won J, Alfini AJ, Weiss LR, Hagberg JM, Smith JC (2020) Greater semantic memory activation after exercise training cessation in older endurance-trained athletes. J Aging Phys Act 29, 250-258.

[60] Kayes MK, Hatfield BD (2019) The influence of physical activity on brain aging and cognition: The role of cognitive reserve, thresholds for decline, genetic influence, and the investment hypothesis. In Lifestyle Medicine, Third Edition, CRC Press, pp. 1251-1269.

[61] Rao SM, Bonner-Jackson A, Nielson KA, Seidenberg M, Smith JC, Woodard JL, Durgerian S (2015) Genetic risk for Alzheimer's disease alters the five-year trajectory of semantic memory activation in cognitively intact elders. Neuroimage 111, 136-146.

[62] Maass A, Berron D, Harrison TM, Adams JN, La Joie R, Baker S, Mellinger T, Bell RK, Swinnerton K, Inglis B (2019) Alzheimer's pathology targets distinct memory networks in the ageing brain. Brain 142, 2492-2509.

[63] Buckner RL, Andrews-Hanna JR, Schacter DL (2008) The brain's default network: Anatomy, function, and relevance to disease. Ann N Y Acad Sci 1124, 1-38.

[64] Buckner RL (2004) Memory and executive function in aging and AD: Multiple factors that cause decline and reserve factors that compensate. Neuron 44, 195-208.

[65] Guo H, Xue J, Cui H, Chen Z, Wang X, Gao Y, Wang B, Xiang J (2019) Altered directed functional connectivity of the hippocampus in mild cognitive impairment and Alzheimer's disease: A resting-state fMRI study. Front Aging Neurosci 11, 326.
[66] Woo C-W, Krishnan A, Wager TD (2014) Cluster-extent based thresholding in fMRI analyses: Pitfalls and recommendations. Neuroimage 91, 412-419.

[67] Firth J, Stubbs B, Vancampfort D, Schuch F, Lagopoulos J, Rosenbaum S, Ward PB (2018) Effect of aerobic exercise on hippocampal volume in humans: A systematic review and meta-analysis. Neuroimage 166, 230-238.

[68] Frerichs RJ, Tuokko HA (2005) A comparison of methods for measuring cognitive change in older adults. Arch Clin Neuropsychol 20, 321-333.

[69] McSweeny AJ, Naugle RI, Chelune GJ, Lüders H (1993) “T scores for change": An illustration of a regression approach to depicting change in clinical neuropsychology. $\mathrm{Clin} \mathrm{Neu}$ ropsychol 7, 300-312.

[70] Lautenschlager NT, Cox KL, Flicker L, Foster JK, Van Bockxmeer FM, Xiao J, Greenop KR, Almeida OP (2008) Effect of physical activity on cognitive function in older adults at risk for Alzheimer disease: A randomized trial. JAMA 300, 1027-1037.

[71] Stott J, Spector A (2011) A review of the effectiveness of memory interventions in mild cognitive impairment (MCI). Int Psychogeriatr 23, 526-538.

[72] Farlow MR (2009) Treatment of mild cognitive impairment (MCI). Curr Alzheimer Res 6, 362-367. 